\title{
Brexit et droits sociaux
}

\section{Catherine Barnard}

\section{(2) OpenEdition \\ Journals}

Édition électronique

URL : https://journals.openedition.org/rdctss/2011

DOI : 10.4000/rdctss.2011

ISSN : 2262-9815

\section{Éditeur}

Centre de droit comparé du travail et de la sécurité sociale

\section{Édition imprimée}

Date de publication : 1 avril 2018

Pagination : 102-114

ISSN : 2117-4350

\section{Référence électronique}

Catherine Barnard, «Brexit et droits sociaux », Revue de droit comparé du travail et de la sécurité sociale [En ligne], 1 | 2018, mis en ligne le 01 novembre 2021, consulté le 13 novembre 2021. URL : http:// journals.openedition.org/rdctss/2011; DOI : https://doi.org/10.4000/rdctss.2011

\section{(c) (i) (9)}

Revue de droit comparé du travail et de la sécurité sociale est mise à disposition selon les termes de la Licence Creative Commons Attribution - Pas d'Utilisation Commerciale - Pas de Modification 4.0 International. 


\section{BREXIT ET DROITS SOCIAUX}

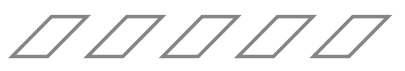

\section{RÉSUMÉ}

Cet article examinera le rôle des droits sociaux de l'Union Européenne au cours de la campagne de référendum, ainsi que ce qu'il adviendra des droits sociaux dans le monde post-Brexit à la lumière du projet de loi (retrait) de l'UE, de l'engagement de non-régression des droits sociaux et de ce que I'UE pourrait exiger dans l'éventualité d'un futur accord commercial. Cet article démontrera que même si l'avenir peut sembler quelque peu sombre pour le maintien des droits sociaux de l'UE, la catastrophe imminente que certains craignent ne se produira pas. Au lieu de cela, il existe un risque de stagnation des droits sociaux existants et une érosion graduelle de la protection dont bénéficient actuellement les salariés, surtout si le Royaume-Uni n'est plus contraint de continuer à respecter les règles du Droit social européen.

MOTS CLÉS : Brexit, droits sociaux, accords commerciaux.

\section{ABSTRACT}

This article will consider the role of EU social rights in the referendum campaign, what will become of social rights in the post Brexit world in the light of the EU (Withdrawal) Bill and the commitment to no-regression of social rights and what the EU might require in the event of a future trade deal. It will argue that while the future may look somewhat bleak for the maintenance of EU social rights, the imminent catastrophe that some fear will not happen. Instead there is a risk of stagnation of existing employment rights and a gradual erosion of the protection employees currently enjoy, especially if there is no robust requirement on the UK to continue to respect EU employment law rules.

KEYWORDS : Brexit, Social Rights, Trade Deals. 


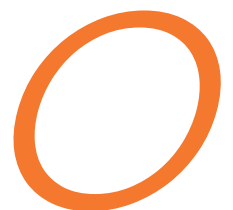

$\mathrm{n}$ aurait pu penser qu'à l'occasion du référendum britannique sur le brexit les préoccupations relatives à la perte des droits sociaux, en cas de vote favorable à la sortie de l'UE, auraient occupé le devant de la scène chez les partisans du "Remain » souhaitant rester européens. Cela n'a pas été le cas. Les droits sociaux n'ont joué qu'un rôle de figuration. Cet article étudiera d'abord pourquoi la question des droits sociaux n'a pas été plus présente dans la campagne référendaire (I). Ensuite, nous examinerons le sort des droits sociaux dans le monde du post-Brexit à la lumière du projet de loi sur le retrait de l'UE (Retrait) et de l'engagement de non-régression des droits sociaux (II). Enfin, nous verrons ce que I'UE pourrait exiger dans le cas d'un futur accord commercial (III). II faut souligner que même si l'avenir peut sembler relativement sombre, l'imminence d'une catastrophe redoutée par certains ne se produira pas. Le risque est plutôt celui d'une stagnation des droits existants et d'érosion graduelle de la protection dont bénéficient actuellement les salariés, en l'absence d'une obligation pour le Royaume-Uni de continuer à respecter les règles du droit du travail de l'UE.

\section{I - POURQUOI LES DROITS SOCIAUX N'ONT-ILS PAS OCCUPÉ UNE PLACE PLUS IMPORTANTE DANS LA CAMPAGNE RÉFÉRENDAIRE ?}

Les partisans que l'on pouvait s'attendre à trouver dans le camp du Remain comme les syndicats se sont en réalité montrés prudents vis-à-vis des arguments invoqués en faveur des droits sociaux de I'UE, sans parler de lutter pour rester dans l'Union. Cela est dû en partie au fait que de nombreux activistes et militants syndicaux soutenaient la campagne pour le "Leave », favorable au retrait. De nombreux défenseurs de cette ligne s'inquiétaient des implications du maintien de l'adhésion à l'Union. Ils craignaient la migration «illimitée » en provenance de l'Union qui, selon eux, a des répercussions négatives sur les salaires. Comme lıa déclaré un responsable syndical après le référendum : « les conditions salariales ont toujours été meilleures pour les travailleurs lorsque la main-d'œuvre est contrôlée et que les communautés sont stables ${ }^{1} »$.

Les syndicalistes s'inquiétaient également des effets négatifs de la mondialisation ainsi que de l'européanisation, et de leur répercussion à l'échelle locale sur le secteur manufacturier. Ils constataient la délocalisation des emplois à l'étranger. Plus généralement, ils s'inquiètent d'une précarisation croissante de l'emploi². La réduction des emplois manufacturiers a coïncidé avec une hausse de l'immigration. L'année du référendum le Royaume-Uni a connu le plus haut niveau d'immigration. Souvent, ces immigrants (plus instruits) ont pourvu des emplois peu qualifiés qui auraient pu être offert aux travailleurs

1 The Guardian, 16 décembre 2016, cité par K. Ewing, « Implications of the Post - Brexit Architecture for Labour Law », Kings Law Journal, 2018, à paraître.

2 Voir M. Taylor, Good Work: The Taylor Review of Modern Working Practices, 2017. 
britanniques récemment contraints au chômage. Cela a renforcé le sentiment de rancœur. Les syndicats ont reconnu que tout convergeait pour rendre les conditions du maintien dans I'UE particulièrement difficiles ${ }^{3}$.

Les syndicats eux-mêmes n'étaient pas unanimement favorables à l'appartenance à I'Union. S'il est vrai que I'UE a accordé de nouveaux droits significatifs aux travailleurs, les droits d'information et de consultation des travailleurs présentent un risque de marginalisation du rôle des syndicats. En outre, les décisions de la Cour de justice dans la lignée de la jurisprudence des affaires (tristement) célèbres Viking et Lava/4 ont laissé un goût amer : ces décisions ont eu pour effet de rendre les actions de grève au RoyaumeUni ayant une composante transnationale encore plus difficiles qu'elles ne l'étaient en vertu d'un droit national déjà strict. Les syndicats ont également vu comment l'Union s'est comportée envers la Grèce pendant la crise financière : les terribles effets des politiques d'austérité et l'abaissement forcé des protections sociales nationales, y compris en matière de négociation collective ${ }^{5}$ ont incité des partis à plaider en faveur d'un départ de l'Union, le « brexit».

Il y a eu aussi un élément tactique. Les syndicats ont reconnu que le fait de faire valoir à plusieurs reprises l'argument selon lequel I'Union était à l'origine de droits sociaux importants faisait en réalité le jeu des partisans du Leave. Les défenseurs de ce camp ont fait valoir que le droit du travail de l'Union était un symbole de la mesure de la perte de contrôle du Royaume-Uni sur « ses propres lois ». Les partisans du Leave pouvaient associer ces préoccupations à des craintes plus générales concernant une réglementation excessive : non seulement ces règles étaient «étrangères», mais elles imposaient également des fardeaux -inutiles- aux entreprises britanniques. Un éminent membre du Parlement, Priti Patel, a résumé cela de la manière suivante : "Si nous pouvions réduire de moitié le fardeau de la législation sociale et de l'emploi de l'Union, nous pourrions injecter un bonus de 4,3 milliards de livres sterling dans notre économie et créer 60000 nouveaux emplois. » Frances O'Grady, secrétaire générale du TUC et l'une des principales personnalités soutenant le Remain a réagi à ce propos avec agressivité: «Quittez I'UE et vous perdez vos droits du travail - c'est le message que même les militants du Leave comme Priti Patel transmettent en ce moment. Quels droits disparaîtraient : le droit aux congés payés, le droit au congé parental, voire les protections pour les travailleuses enceintes? L'UE garantit tous ces droits et bien davantage ; c'est la raison pour laquelle le Brexit représente un si grand

3 http://blog.policy.manchester.ac.uk/posts/2017/08/trade-unions-eu-workers-and-brexit-morecomplexity-less-certainty/

4 Aff. C-438/05, International Transport Workers' Federation and Finnish Seamen's Union v Viking Line ABP[2007] ECR I-10779 etAff. C-341/05, Laval un Partneri Ltdv Svenska Byggnadsarbetareförbundet, Svenska Byggnadsarbetareförbundets avdelning 1, Byggettan and Svenska Elektrikerförbundet [2007] I-11767. Pour une discussion sur les effets corrosifs de la décision sur le droit du travail britannique, voir C. Barnard, "British Jobs for British Workers': The Lindsey Oil Refinery Dispute and the Future of Local Labour Clauses in an Integrated EU Market', ILJ, 2009, n³8, p. 245.

5 https://www.theguardian.com/politics/2016/jun/21/the-labour-case-for-a-leave-vote-in-the-eureferendum 
risque pour les travailleurs ». Dix secrétaires généraux des plus grands syndicats ont soutenu les propos de la secrétaire générale du TUC et ont appelé à voter en faveur du Remain ${ }^{6}$. Nonobstant, cette intervention s'est avérée insuffisante et surtout trop tardive.

\section{II - LE SORT DES DROITS SOCIAUX APRÈS LE BREXIT}

Après le vote en faveur du départ de l'Union, on s'attendait à ce que la règlementation nationale sur le temps de travail de transposition de la directive sur le temps de travail, une des lois de l'UE les plus contestées par les employeurs', soient rapidement abrogée après le Brexit, et que dans la foulée, il en aille de même des lois sur le travail intérimaire et le travail à durée déterminée ${ }^{8}$. Ce fut donc une surprise lorsque la Première ministre a annoncé que les droits sociaux seraient maintenus dans le monde de l'après-Brexit. Dans son discours de la conférence du parti en octobre 2016, elle a déclaré : "En abrogeant la loi sur les Communautés européennes, nous transformerons les acquis... dans le cadre du droit britannique. Lorsque le projet de loi sur l'abrogation [ou EU(W)B] recevra l'approbation royale, le Parlement sera libre - sous réserve des accords internationaux et des traités avec d'autres pays et l'UE en matière de commerce notamment - de modifier, abroger et améliorer toutes les lois. Mais en transférant les acquis dans le droit britannique, nous donnerons aux entreprises et aux travailleurs un maximum de garanties au moment où nous quitterons I'Union européenne.... Et je tiens à être parfaitement claire: les droits légaux existants des travailleurs continueront d'être garantis par la loi - ils le seront tant que je serai Premier ministre $»$.

Cet engagement politique a été réitéré dans le livre blanc sur le Brexit : ${ }^{10}$ " $A$ mesure que nous transposerons le droit communautaire dans notre législation nationale, nous assurerons la continuité de la protection des droits des travailleurs. Cela apportera des garanties et assurera une certaine continuité aux salariés et aux employeurs qui engendrera une stabilité qui permettra au Royaume-Uni de poursuivre sa croissance et sa prospérité ».

Plus récemment, dans son discours de Mansion House, la Premier ministre a déclaré :11

"Et dans d'autres domaines tels que les droits des travailleurs ou l'environnement, l'UE doit être convaincue que nous ne nous engagerons pas dans un nivellement par le bas en

6 Les syndicats britanniques incitent à voter contre le Brexit.

https://www.politico.eu/article/eu-referendum-leave-remain-uk-trade-unions-brexit-news-ukpolitics/. Leurs lettres ont été publiées dans le Guardian

https://www.theguardian.com/politics/2016/jun/05/trade-union-members-should-vote-to-stay-inthe-eu

7 Sunday Times, 17 décembre 2017; Sun on Sunday, 17 décembre 2017. Pour une analyse plus approfondie, voir Huffington Post, 19 décembre 2017, cités par K. Ewing, ci-dessus note 1.

8 Voir généralement M Ford, "The Effect of Brexit on Workers' Rights", King's Law Journal, 2016, n²7, p. 398.

9 Extrait du discours souligné par nous

10 Gouvernement britannique, The United Kingdom's exit from and new partnership with the European Union (Cm 9417, February 2017)

11 https://www.gov.uk/government/speeches/pm-speech-on-our-future-economic-partnershipwith-the-european-union 
ce qui concerne les normes et les protections que nous établirons. Aucun groupe politique sérieux au Royaume-Uni ne soutiendrait cela - bien au contraire ».

En réalité, au sein même du Cabinet, des personnalités s'opposent aux droits du travail. Par exemple, Liam Fox, le secrétaire d'État au Commerce international, a déclaré : "Pour rétablir la compétitivité de la Grande-Bretagne, nous devons commencer par déréglementer le marché du travail. Les oppositions politiques doivent être surmontées ${ }^{12}$ ». Cependant, tant que Theresa May demeurera Premier ministre, les droits du travail semblent relativement assurés; le langage de la non-régression commence, y compris, à être repris. Mais les droits sociaux sont-ils réellement assurés ? Pour le moment, la garantie la plus importante de la protection des droits du travail réside dans le projet de loi (de retrait) de I'UE (EU (W) B).

\section{A - LE MAINTIEN DES LOIS DE L'UE}

Le projet de loi (de retrait) de l'UE, actuellement examiné par le Parlement, vise à sortir le Royaume-Uni de l'UE au niveau national tout en garantissant la sécurité et la continuité juridiques au lendemain du Brexit, y compris dans le cas où il n'y aurait aucun accord avec I'Union. Plus précisément, l'objectif est de prendre un instantané du droit de l'UE tel qu'il se présente au moment du Brexit et de le transposer dans la législation nationale. Ainsi, l'article 2 (1) prévoit que "la législation nationale dérivée de l'Union, ayant un effet sur le droit national juste avant la sortie de l'UE, continuera d'avoir un effet sur le le droit national à la date de sortie et après ». La législation nationale dérivée de l'UE comprend la législation adoptée en vertu des pouvoirs énoncés au paragraphe 2 de liarticle 2 de la loi de 1972 sur les Communautés européennes (ECA), la loi qui, à l'origine, a entériné l'entrée du RoyaumeUni dans I'UE. L'article 2 (2) est une « clause Henry VIII » (du nom du célèbre roi britannique) qui permet à l'exécutif de mettre en œuvre la législation européenne comme législation secondaire sans passer par le processus intégral d'adoption d'une loi par le Parlement. Ainsi, la règlementation sur le temps de travail, qui met en œuvre la directive y relative, a été mise en œuvre en vertu des pouvoirs conférés par le paragraphe 2 (2) et restera inscrite dans la législation britannique en vertu de l'article 2 de l'UE (W)B). L'article 2 de l'UE (W) B sauvegardera également d'autres textes de loi adoptés en vertu d'autres pouvoirs, tels que les lois sur le travail à durée déterminée prise en vertu de la loi de 2002 sur l'emploi. La législation primaire donnant effet à la législation de l'UE à l'instar de la loi de 2010 sur l'égalité restera également en vigueur. Ce droit est dénommé "droit de l'UE maintenu » (article 6, paragraphe 7, UE (W) B) et pourra faire l'objet de modifications ultérieures par le Parlement (sauf accord contraire futur).

Ainsi, l'article 2 de l'UE (W) B prévoit le mécanisme juridique garantissant que les droits sociaux de l'UE resteront en vigueur après le départ du Royaume-Uni, que ce soit le 29 mars 2019 s'il n'y a pas d'accord, ou le 31 décembre 2020 à la fin de la période de transition (en vertu de la partie IV de l'accord de retrait, la législation de l'UE continuera à sıappliquer au Royaume-Uni comme s'il était un Etat membre pendant la période de transition). Jusque-là, tout est simple. Cependant, toutes les lois de l'Union ne peuvent pas être conservées dans leur format actuel. Prenons, par exemple, le Règlement de 1999 relatif à l'information et la

12 https://www.ft.com/content/2ee5b8de-5c8d-11e1-8f1f-00144feabdc0\#axzz1 mtrhfavw 
consultation des travailleurs $(\text { TICE })^{13}$ et modifié en $2010^{14}$. Les règlements ont été adoptés en vertu des pouvoirs énoncés au paragraphe 2 (2) de la loi de 1972 sur les Communautés européennes, exigeant qu'une société multinationale ou, selon le jargon européen, une "entreprise de dimension communautaire ", crée un comité d'entreprise européen ou un équivalent aux fins de donner des informations aux représentants des travailleurs et de les consulter. Cependant, la définition d'une entreprise de dimension communautaire selon la législation du Royaume-Uni désigne une entreprise ayant « au moins 1000 salariés dans les Etats membres et au moins 150 dans chacun d'au moins deux Etats membres ». Lorsque le Royaume-Uni ne sera plus un Etat membre, se posera un problème.

\section{B - REMÉDIER AUX LACUNES DU DROIT BRITANNIQUE}

L'article 7 du projet de loi confère au gouvernement le pouvoir de faire face aux soidisant « lacunes » de la loi actuelle lorsque le pouvoir exécutif estime qu'il est « approprié » de le faire. Cependant, ces soi-disant « pouvoirs Henry VIII » sont controversés parce qu'ils sont exceptionnellement étendus ${ }^{15}$ et permettent au gouvernement de modifier les deux instruments statutaires (SI) (loi secondaire) et les lois du Parlement (loi primaire), donnant ainsi « aux ministres une bien plus grande latitude que ce qui est constitutionnellement acceptable ${ }^{16} »$. Au cours des débats au sein de la Chambre des communes, le gouvernement a cependant admis qu'il faudrait une nouvelle commission parlementaire chargée d'examiner les instruments statutaires introduits pour modifier le droit de l'UE retenu. Le comité décidera si ces IS doivent faire l'objet d'une procédure négative ou positive (cette dernière devant être approuvée par les deux chambres du Parlement). La Chambre des lords, pour sa part, ne considère pas ces garanties suffisantes et en exige davantage.

Néanmoins, l'article 7 sera maintenu sous une forme ou une autre et le Royaume-Uni pourrait décider d'utiliser les pouvoirs de l'article 7 pour supprimer le règlement 4 (1) du TICE sur les entreprises de dimension communautaire dont le siège social se trouve au Royaume-Uni et il pourrait supprimer le règlement 5 sur le siège social ou l'instance de représentation représentatif située au Royaume-Uni. Les syndicats espèrent que, conformément au principe de non-régression, les dispositions du règlement TICE relatives à la protection de l'emploi ne seront pas abrogées. Cependant, tout cela fera l'objet d'un accord entre le Royaume-Uni et l'UE comme nous le verrons.

Bien que l'article 7 habilite le ministre de la Couronne à empêcher, réparer ou atténuer: (a) tout empêchement au bon fonctionnement du droit européen maintenu, ou (b) toute autre déficience du droit de l'UE résultant du retrait du Royaume-Uni de l'UE, il est à craindre qu'un ministre utilise ces pouvoirs pour apporter des changements plus substantiels sous prétexte de remédier à une lacune. Des craintes particulières portent sur les dispositions de la loi de 2010 sur l'égalité qui risquent d'être menacées. Il existe donc une pression pour ajouter la Loi sur l'égalité à la liste établie par la clause 7 (7) selon laquelle les pouvoirs du

13 http://www.legislation.gov.uk/uksi/1999/3323/introduction/made

14 http://www.legislation.gov.uk/uksi/2010/1088/contents/made

15 https://publications.parliament.uk/pa/ld201719/ldselect/ldconst/19/1906.htm

16 https://publications.parliament.uk/pa/ld201719/ldselect/ldconst/69/6903.htm\# idTextAnchor003 
paragraphe 7 (1) ne peuvent pas être utilisés dans certains domaines, notamment pour modifier, abroger ou révoquer la Loi sur les droits de la personne de 1998 qui intègre la Convention européenne des droits de l'homme dans la législation britannique.

\section{C - LA CHARTE DES DROITS FONDAMENTAUX}

Bien que l'idée clé qui sous-tend l'UE (W) B soit celle de la poursuite, et non de la rupture, au moins au départ, l'exception notable porte sur la Charte des droits fondamentaux. L'article 5 (4) stipule que : "La Charte des droits fondamentaux ne fait pas partie du droit interne au moment de la sortie de I'UE ou après. " Toutefois, cela n'affecte pas le maintien dans le droit interne, des droits ou principes fondamentaux qui existent indépendamment de la Charte. En outre, le texte précise que les références à la Charte dans la jurisprudence sont autant que nécessaire, interprétées comme sıil sıagissait de références à des droits ou principes fondamentaux correspondant ayant été conservés. Par conséquent, alors que la Charte ne sera pas conservée après le Brexit, les principes généraux qu'elle consacre le seront. L'annexe I, article 2 fournit des détails supplémentaires. Elle prévoit qu'aucun principe général de droit européen ne fait partie du droit interne à la date de sortie ou après, s'il n'a pas été reconnu comme principe général de droit européen par la Cour européenne dans une affaire judiciaire résolue avant la date de sortie (qu'il s'agisse ou non d'une partie essentielle de la décision dans l'affaire en cause). De plus, contrairement aux droits découlant de la Charte, où une action en dommages-intérêts peut être intentée en cas de violation, l'article 3 de l'annexe I stipule que : « II n'y a pas de droit d'action en droit national le jour de la sortie ou par la suite sur la base d'un non-respect de l'un quelconque des principes généraux du droit de l'UE. » En outre, aucune Cour, aucun tribunal ou aucune autorité publique ne peut, le jour de la sortie de l'UE ou après 1. Réformer, abroger toute loi ou toute autre règle de droit, ou 2. Censurer une conduite quelle qu'elle soit ou décider qu'elle est illégale, parce qu'elle est incompatible avec l'un des principes généraux du droit de I'UE.

Pour faire bonne mesure, l'article 4 ajoute : « II n'y a pas droit à des dommages-intérêts conformément à l'arrêt Francovich en droit national, que ce soit le jour de la sortie, ou par la suite. »Par conséquent, non seulement la Charte disparaît, mais certains des principaux recours disparaissent aussi, en particulier les arrêts apports des Francovich et Mangold.

Qu'en est-il de la perte des droits de la Charte ? Les avocats ont été quelque peu ambigus au sujet de la Charte des droits fondamentaux ${ }^{17}$. Alors que le titre IV sur la solidarité était très prometteur, son contenu et son application ont été en réalité décevants ${ }^{18}$. Dans l'affaire, peut-être la plus controversée, Alemo-Herron ${ }^{19}$, une référence des tribunaux britanniques sur l'interprétation de la mise en œuvre plus généreuse au Royaume-Uni de la directive sur le transfert des entreprises, la Cour s'est appuyée sur le droit de direction une entreprise en vertu de l'article 16 pour contester et supprimer cette protection parce

17 C. Barnard, 'The Silence of the Charter' dans S De Vries, U Bernitz et S Weatherill (eds), The EU Charter of Fundamental Rights as a Binding Instrument: Five Years Old and Growing, Hart Publishing, Oxford 2015.

18 Affaire C-426/11, Alemo-Herron v Parkwood Leisure, ECLI:EU:C:2013:521; Affaire C-201/15, AGET Iraklis, ECLI:EU:C:2016:972.

19 Alemo-Herron and Others v Parkwood Leisure Ltd, ci-dessus n 17, paragraphes 31-35. 
qu'elle était réputée porter atteinte à la liberté de direction de l'employeur. A aucun moment, la Cour n'a considéré l'application de l'une quelconque des dispositions du titre sur la solidarité comme un contrepoids au droit de l'employeur garanti par l'article 16. Les droits conférés par le titre sur l'égalité ont toutefois été plus prometteurs et ont été utilisés au profit des travailleurs ${ }^{20}$, bien qu'ici, au moins, le principe général d'égalité ait une origine plus ancienne et mieux établie.

\section{D - LE RÔLE DES TRIBUNAUX}

L'article 1 de l'UE (W) B abroge l'ECA 1972, mettant fin aux principes de suprématie et d'effet direct dans le système juridique britannique (principes qui devront être ré-établis pendant la période de transition, ce qui sera établi par une autre loi britannique sur le retrait et la mise en œuvre dite WAIB). Cependant, la situation n'est pas aussi claire qu'il y paraît. L'article 5 prévoit que si le principe de la suprématie du droit de l'Union ne s'applique pas aux textes promulgués après le Brexit, il s'applique néanmoins pour " l'interprétation, la non-invocation ou l'annulation de toute loi ou règle de droit adoptée avant la sortie ". Cela signifie donc que si les tribunaux britanniques constatent dans une affaire que la loi de 2010 sur l'égalité - adoptée avant le Brexit - contient des dispositions contraires, par exemple, à l'article 157 TFUE, alors l'article 157 TFUE prévaudra. La principale différence est qu'après le Brexit, le Royaume-Uni sera libre de légiférer pour annuler les effets d'une telle solution (sous réserve de tout accord commercial à venir)

Qu'en est-il de la pertinence de la jurisprudence de la Cour de justice ? Concernant celle antérieure au Brexit, elle continuera à s'appliquer en tant que loi britannique (comme la loi britannique, bien que, en droit britannique, elle puisse être annulée par la Cour suprême du Royaume-Uni).. ${ }^{21}$ Cependant, la position de la jurisprudence post-Brexit est plus compliquée. L'article 6 prévoit qu'une cour ou un tribunal : a) n'est lié par aucun des principes énoncés ou des décisions prises par la Cour européenne le jour de la sortie ou après, et b) ne peut saisir la Cour européenne le jour de la sortie ou après (bien entendu, il n'y aura pas non plus d'actions coercitives au titre de l'article 258 du TFUE). De plus, la clause 6 (2) stipule que: "Une cour ou un tribunal n'a aucune obligation de tenir compte de ce qui a été fait au jour de la sortie ou après par la Cour européenne de justice, une autre entité de l'UE ou l'UE ; il peut le faire si cela lui semble approprié ».

Ainsi, après le Brexit, les tribunaux britanniques ne sont pas liés par les arrêts de la Cour de justice, ils ne peuvent pas non plus faire référence à l'article 267, mais ils peuvent prendre en compte ces décisions s'ils les jugent « appropriées ». Les juges sont profondément inquiets du pouvoir que cela leur donne. Ils craignent que la décision de prendre en compte les décisions de la CJCE ne devienne politique et non juridique et considèrent qu'elles seront en première ligne des attaques médiatiques. Ils souhaiteraient que le libellé de cette disposition soit modifié.

Il est cependant possible que ces préoccupations soient exagérées, au moins dans le domaine de l'emploi²2. Les juges britanniques n'ont jamais suivi servilement les arrêts de la

20 Affaire C176/12, Association de médiation sociale, ECLI:EU:C:2014:2.

21 Articles 6(4) et (5) EU(W)B.

22 C. Barnard, «Brexit and Employment law», in M. Dougan (dir.), The UK after Brexit, Intersentia, 2017. 
Cour de justice, surtout lorsqu'ils ne les jugent pas raisonnables. Un bon exemple est celui de l'affaire $E C M^{23}$, dans laquelle la Cour d'appel a adopté une ligne pragmatique pour gérer les contestations de la décision très critiquée de la Cour de justice Süzen ${ }^{24}$ pour déterminer si un transfert d'entreprise avait eu lieu dans le cas d'une entreprise employant beaucoup de main-d'œuvre. La décision de la Cour de justice a utilisé des critères permettant de savoir si la majorité du personnel avait été transféré pour déterminer s'il y avait eu un transfert d'entreprise qui aurait déclenché le transfert de ces mêmes salariés. En d'autres termes, le raisonnement était circulaire. La Cour d'appel a préféré l'approche Smorgasbord adoptée par la Cour de justice dans l'affaire Spijkers plus ancienne ${ }^{25}$, qui consistait à examiner une série de facteurs, pour déterminer si un transfert d'entreprise avait eu lieu et donc si ce personnel devait être transféré.

Dans l'affaire Govia ${ }^{26}$, la Cour d'appel a aussi rogné les griffes de la décision controversée de la Cour de justice dans l'affaire Viking ${ }^{27}$. Viking a eu des conséquences néfastes sur le droit de grève au Royaume-Uni ${ }^{28}$. Dans l'affaire Govia, la Cour d'appel a dû examiner si la grève proposée par les syndicats à propos de l'introduction de trains de voyageurs uniquement gérés par des conducteurs (DOOP) violait l'article 49 TFUE, l'argument étant qu'une société française, Keolis, détenait 35\% des actions de Govia et que la grève entravait l'exercice des droits tirés de la liberté d'entreprise. La Cour d'appel a déclaré: 39....[C'] est l'objet ou le but de l'action collective et non le dommage causé par l'action elle-même qui la soumet potentiellement aux dispositions relatives à la liberté de mouvement. ... A notre avis, il est inconcevable qu'une règle qui ne fait pas de discrimination fondée sur la nationalité et qui oblige un conducteur et un gardien sur tous les trains à assurer la fermeture des portes plutôt que cela soit un conducteur seul puisse constituer un facteur entravant la liberté d'entreprise ou le rendre moins attrayant. »

La Cour d'appel a ensuite souligné que, même lorsqu'une mesure était appliquée au détriment d'une personne, cela ne permettait pas de déduire pour autant que sa liberté de circulation a été violée si l'effet est trop incertain, indirect ou insignifiant pour avoir l'effet dissuasif requis. La Cour a conclu en ces termes « Nous nıavons aucun doute sur le fait qu'en l'espèce c'était trop insignifiant ».

II se peut donc que les tribunaux britanniques, après le Brexit, se sentent plus libérés et capables d'en faire plus dans ce sens, et qu'ils façonnent la jurisprudence post-Brexit pour mieux s'adapter aux circonstances du marché du travail britannique, y compris en ce qui concerne les questions controversées telles que le paiement des périodes de congés payés $^{29}$ ( par exemple, les contrats à court terme incluant le paiement des congés payés,

23 ECM v Cox [1999] IRLR 559.

24 Aff. C-13/95, Süzen v Zehnacker Gebäudereinigung GmbH, ECLI:EU:C:1997:141.

25 Aff. 24/85, Spijkers v Benedik, ECLI,EU,C,1986,127.

26 Govia v The Associated Society of Locomotive Engineers [2016] EWCA (Civ) 1309.

27 Aff. C-438/05, International Transport Workers' Federation and Finnish Seamen's Union v Viking Line ABP and OÜ Viking Line Eesti, ECLI, EU, C:2007,772.

28 Voir C. Barnard, "The Calm after the Storm. Time to Reflect on EU Scholarship after Viking and Laval" in A Bogg, C Costello and A Davies (dir.), Research Handbook on EU Labour Law, Elgar, Cheltenham, 2016.

29 https://www.gov.uk/holiday-entitlement-rights/holiday-pay-the-basics 
une solution pragmatique jugée illégale par la Cour de justice ${ }^{30}$ ). A cet égard, l'expérience suisse est pleine d'enseignement. Officiellement, les arrêts de la Cour de justice n'ont qu'un caractère persuasif. Pendant des années, cela signifiait qu'un jugement suisse équivalant était nécessaire pour expliquer l'effet de l'arrêt de la Cour de justice en Suisse. Comme cela devenait ingérable, la Cour suprême suisse a décidé en 2009 que les arrêts de la Cour de justice s'appliqueraient à moins qu'il n'y ait une bonne raison pour qu'ils ne le soient pas $^{31}$. En réalité, les juridictions suisses appliquent assez fidèlement les arrêts de la Cour de justice. Si elles ne le font pas, reconnaissent-elles, le droit suisse ne sera pas à jour par rapport au droit de l'UE. Chose importante, à la lumière d'un futur accord commercial entre I'UE et le Royaume-Uni, les Suisses reconnaissent que le non-respect peut également mettre en péril les relations commerciales de la Suisse avec lıUE.

Enfin, qu'en est-il en cas d'une véritable incertitude sur la signification du droit de l'UE en l'absence de la procédure de renvoi prévue à l'article 267 TFUE ? Le recours à un comité mixte UE-Royaume-Uni pourrait être une solution; mais ce comité pourrait ne pas avoir de juges dans son panel. Compte tenu de la réticence générale des tribunaux britanniques à saisir la CJUE, il se peut que les juges britanniques en tirent le meilleur parti et décident eux-mêmes du sort des affaires. Des décisions comme Govia et ECM montrent que les résultats ne sont pas nécessairement mauvais pour les salariés.

\section{III - L'AVENIR}

Dans son discours de Lancaster House ${ }^{32}$, Theresa May a déclaré que le gouvernement britannique s'appuierait sur les droits consacrés par la législation européenne ${ }^{33}$. Le livre blanc sur le Brexit développe cette idée et précise que ces dernières années, le gouvernement a pris des mesures indépendantes pour protéger les travailleurs britanniques et assurer leur traitement équitable. Dans de nombreux domaines, le gouvernement britannique a déjà étendu les droits des travailleurs au-delà de ceux énoncés dans le droit de l'UE. Elle cite, à titre d'exemple, le fait que:

- La loi nationale -britannique- prévoit déjà 5,6 semaines de congé payé annuel, alors que le droit de l'UE n'en prévoir que 4.

- Au Royaume-Uni, les femmes qui ont eu un enfant peuvent bénéficier de 52 semaines de congé légal de maternité, dont 39 semaines avec maintien du salaire, et pas seulement 14 semaines comme dans le droit de l'UE,

30 Aff. C-131/04, C D Robinson-Steele v R D Retail Services Ltd, ECLI,EU, C, 2006,177.

31 Jugement du tribunal fédéral suisse (136 || 5), discuté dans T. Burri, "Do Lawyers Knead the Dough? How Law, Chaos and Uncertainty Interact" European Journal of Risk Regulation, 2010, $n^{\circ} 4$, p. 371. Voir également T. Burri, "Brexit addendum: Why the "Great Repeal Bill" will not be so great", https://papers.ssrn.com/sol3/papers.cfm?abstract id=2966683.

32 https://www.gov.uk/government/speeches/the-governments-negotiating-objectives-for-exitingthe-eu-pm-speech

33 Voir également les engagements dans le manifeste conservateur Conservative Party, Forward Together: The Conservative Manifesto, 2017, par exemple à la page 7. 
- Le Royaume-Uni offre une plus grande flexibilité en ce qui concerne le congé parental partagé ; dans certaines conditions, le père peut partager le congé parental, ce qui donne aux familles le choix d'équilibrer leurs responsabilités domestiques et professionnelles,

- Le Royaume-Uni offre un congé parental de 18 semaines; cela est plus favorable que la Directive de l'UE sur le sujet car le travailleur peut en jouir jusqu'au $18^{\mathrm{ème}}$ anniversaire de l'enfant ${ }^{34}$.

Selon le livre blanc le gouvernement s'est engagé également à « renforcer les droits quand il sıagira d'un bon choix pour les travailleurs britanniques et continuera à rechercher des opportunités pour renforcer les protections ${ }^{35}$ ». On souligné le fait qu'en avril 2016, le gouvernement a introduit le salaire national de subsistance ainsi que des sanctions plus sévères pour les employeurs délibérément rétifs à se mettre en conformité36.

Tout cela est vrai. Aucun de ces développements n'a été exigé par la législation de l'UE ni contraint par elle. La législation européenne sur l'emploi fixe des normes minimales, et non maximales, et constitue, en tout cas, un régime juridique incomplet. II existe des pans entiers de la politique sociale qui ne sont pas concernés par le droit de l'UE ${ }^{37}$. Les Etats membres ont une liberté considérable de faire ce qu'ils veulent dans le domaine social, comme l'a d'ailleurs montré le Royaume-Uni tout en étant membre de l'UE. Le Brexit en soi ne crée pas d'opportunités pour développer une politique sociale indisponible avant le référendum.

Plus remarquable est la déclaration du Livre blanc selon laquelle, « nous nous sommes engagés à maintenir notre statut de leader mondial des droits des travailleurs et nous veillerons à ce que la protection juridique des travailleurs suive l'évolution du marché du

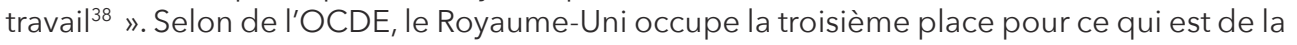
législation du travail (score de 1,10 en 2013) dans le sens où elle a le moins de prescriptions en matière de licenciements individuels et collectifs (après les Etats-Unis (0,26 en 2013) et le Canada $\left(0,92\right.$ en 2013 $\left.{ }^{39}\right)$, un point que le Royaume-Uni a noté avec fierté dans sa Consultation Ending the Employment Relationship ${ }^{40}$. En outre, le Royaume-Uni continue à flexibiliser sa législation du travail, notamment en allongeant l'ancienneté nécessaire pour pouvoir contester en justice un licenciement abusif, en ajoutant les cas d'exclusion du droit porter plainte pour licenciement abusif et, surtout, en imposant des frais d'accès

34 The United Kingdom's exit from and new partnership with the European Union..

$35 \mathrm{lbid}$, paragraphe 7.3.

36 Ibid, paragraphes 7.4-7.5.

37 Voir généralement J. Kenner, EU Employment Law. From Rome to Amsterdam and Beyond, Hart Publishing, Oxford, 2003.

38 The United Kingdom's exit from and new partnership with the European Union, op.cit.

$39 \mathrm{http}: / /$ stats.oecd.org/Index.aspx? DataSetCode=EPL OV $>$. Le chiffre moyen de l'OCDE était de 2,04.

40 BIS, Ending the Employment Relationship Consultation, 2012

https://www.gov.uk/government/uploads/system/uploads/attachment data/file/32700/12-1037ending-the-employment-relationship-consultation.pdf, p 13 
aux tribunaux du travail. Les plaintes ont chuté de $70 \%{ }^{41}$ jusqu'à ce que la Cour suprême déclare la règle illégale ${ }^{42}$. C'est certes une forme de leadership; mais la plupart des juristes en droit travail ne considèrent pas que cela fasse du pays un leader mondial en matière de protection des travailleurs.

De plus, il est frappant de constater que ces développements se sont produits dans des domaines dans lesquels (licenciement abusif et accès aux tribunaux), le Royaume-Uni n'est pas contraint par un plancher de protection prévu par la législation européenne. Cela soulève la question de savoir ce qui se passera quand Theresa May ne sera plus Premier ministre (ou si les négociations se passent mal). Le droit du travail, dans les mains d'un gouvernement conservateur, s'orientera-t-il en fait vers la déréglementation comme Liam Fox et Priti Patel le préconisent, ou du moins vers un processus continu de sape des droits (par exemple réduire le champ d'application personnel de la protection sociale ou augmenter l'ancienneté requise pour qu'un salarié puisse profiter de ce droit)?

Dans son discours de Lancaster House, le Premier ministre a également évoqué la protection juridique des travailleurs qui suivent le rythme de l'évolution du marché du travail. Elle a ensuite abordé la représentation des travailleurs dans les conseils d'administration des entreprises. Cela traduit une approche plus progressiste du droit du travail (bien que cette proposition se soit déjà heurtée à des difficultés) ${ }^{43}$, tout comme la référence ultérieure à l'amélioration des droits des travailleurs. Mais que se passera-t-il si l'économie connaît une récession après le Brexit ? De tels changements sur le marché du travail représententils en fait une déréglementation des droits des travailleurs? Ce risque est exacerbé si aucun accord commercial n'est conclu avec l'UE. Concernant un accord commercial avec I'UE, les orientations politiques publiées le 23 mars 2018 indiquent que ${ }^{44}$ : Compte tenu de la proximité géographique et de l'interdépendance économique du Royaume-Uni avec l'Union des 27, la relationfuture ne sera satisfaisante que si elle comporte de solides garanties solides de maintien de conditions de concurrence équitables. L'objectif devrait être de prévenir les avantages concurrentiels injustes dont le Royaume-Uni pourrait pâtir en réduisant les niveaux de protection en ce qui concerne notamment la concurrence et les aides publiques, ainsi que l'environnement et les mesures règlementaires en matière fiscale et sociales. Cela nécessitera une combinaison de règles substantielles alignées sur les normes européennes et internationales, des mécanismes adéquats pour assurer une mise en œuvre efficace au niveau national, des mécanismes d'exécution et de règlement des différends dans l'accord ainsi que des recours autonomes de l'Union en rapport avec la

41 Voir par exemple "Unions blame 70\% fall in employment tribunal cases on fees"', The Guardian, 31 janvier 2017

https://www.theguardian.com/money/2017/jan/31/employment-tribunal-cases-down-70-sincefees-introduced. See further A. Adams and J. Prassl, "Vexatious Claims? Challenging the Case for Employment Tribunal Fees", Modern Law Review, 2017, n80, p. 412.

$42 R$ (on the application of UNISON) (Appellant) $v$ Lord Chancellor [2017] UKSC 51.

43 'Plans to put workers on company boards falters', Financial Times, 31 octobre 2016

https://www.ft.com/content/22128636-9ece-11e6-891e-abe238dee8e2; bien que Sports Direct ait décidé d'inclure des travailleurs dans le conseil, Financial Times,

https://www.ft.com/content/d4d713ca-04ab-11e7-ace0-1ce02ef0def9

Cependant, l'engagement est réitéré dans le manifeste conservateur de 2017

https://s3.eu-west-2.amazonaws.com/manifesto2017/Manifesto2017.pdf.

44 http://www.consilium.europa.eu/media/33458/23-euco-art50-guidelines.pdf, paragraphe 12. 
profondeur et l'étendue des liens existant entre I'UE et le Royaume-Uni.

Cela soulève autant de questions que de réponses. II est clair que l'UE souhaite que le Royaume-Uni reste proche de son droit social, mais sans préciser si cela implique de continuer à respecter l'acquis existant et l'acquis social futur ou si cela consiste à respecter les normes fondamentales de l'OIT. Et, en l'absence d'accord commercial avec I'UE exigeant des normes sociales élevées, les conséquences pourraient être graves. Comme le dit Ewing ${ }^{45}$, I"ordre du jour de la droite [au Royaume-Uni] est que des accords bilatéraux de libre-échange remplacent l'adhésion du Royaume-Uni à ce qui est le plus grand accord de libre-échange au monde. Ce faisant, ils font la promotion du libre-échange, pas du commerce équitable, des marchés libres et non des marchés sociaux. Il en résultera un nouvel ordre juridique, qui inclura différents types de contestations constitutionnelles et de normes du travail, mais qui n'imposera aucune restriction à l'érosion du droit du travail.

\section{Conclusion}

Les travailleurs britanniques ont beaucoup bénéficié de la protection apportée par le droit social européen et de liapplication de ces droits par la Commission et la Cour de justice. En l'absence d'un accord futur qui imposerait un alignement sur les droits sociaux de I'UE, les travailleurs britanniques restent vulnérables face aux changements d'attitude possibles du Parlement de Westminster en matière de protection des travailleurs. Le droit social international est faible : la Charte sociale européenne et l'OIT offrent une protection nettement plus faible que le droit de I'UE et les recours disponibles pour les travailleurs sont extrêmement faibles. Malgré l'ambivalence des membres des syndicats vis-à-vis de I'UE, en l'absence d'un gouvernement politiquement favorable, ils continueront à dépendre directement ou indirectement de I'UE pour améliorer les normes du travail.

45 K. Ewing, ci-dessus note 1.

\section{CATHERINE BARNARD}

Professeure de Droit de l'Union Européenne et de Droit Social, Trinity College, Université de Cambridge.

Thèmes de recherche : Droit de l'Union Européenne, droit social, droit des discriminations.

\section{Publications :}

C. Barnard, EU Employment Law, Oxford, OUP, 2012.

C. Barnard et A. Ludlow, "The Enforcement of Rights by EU-8 migrants before Employment Tribunals », Industrial Law Journal, Vol. 45, n²1, 2016. 